\title{
Coherent Control and Polarization Readout of Individual Excitonic States
}

\author{
B. Patton, ${ }^{1}$ U. Woggon, ${ }^{1}$ and W. Langbein ${ }^{2}$ \\ ${ }^{1}$ Fachbereich Physik, Universität Dortmund, Otto-Hahn-Str. 4, 44227 Dortmund, Germany \\ ${ }^{2}$ Department of Physics and Astronomy, Cardiff University, Cardiff CF24 3YB, United Kingdom
}

(Received 7 July 2005; published 21 December 2005)

\begin{abstract}
We present measurements and simulations of coherent control and readout of the polarization in individual exciton states. The readout is accomplished by transient four-wave mixing detected by heterodyne spectral interferometry. We observe Rabi oscillations in the polarization, which show half the period of the Rabi oscillations in the population. A decrease of the oscillation amplitude with pulse area is observed, which is not accompanied by a change in the dephasing time. This suggests the transfer of the excitation to other states as the origin of the Rabi-oscillation damping. Detuning of the excitation enables the control of the polarization in phase and amplitude and is in qualitative agreement with simulations for a two-level system. Additionally, simultaneous Rabi flopping of several spatially and energetically close exciton states is demonstrated.
\end{abstract}

DOI: 10.1103/PhysRevLett.95.266401

PACS numbers: 71.35.- y, 42.60.Da, 42.65.Hw

The coherent control and detection of spin states via nuclear magnetic resonance (NMR) was first demonstrated more than 50 years ago [1]. Shortly thereafter, pulsed techniques were developed to control spin systems, and to measure decoherence and dissipation rates. Applying radio-frequency pulses defined by $\pi$ or $\pi / 2$ pulse areas, a spin state can be switched in phase and orientation. This quantum state manipulation resulted in many proof-ofprinciple demonstrations of quantum algorithms by liquid state NMR. While conventional NMR approaches build on well-developed experimental capabilities to create and control the quantum properties necessary for quantum computation in ensembles, solid-state approaches towards coherent control can draw on existing nanostructure fabrication technologies for the addressing of individual qubits. By combining the coherent control techniques of NMR with ultrafast pulses in the optical frequency range, engineered exciton and spin-based scalable quantuminformation processing (QIP) devices are expected to be realizable. The subpicosecond time scale of the optical pulses allows for the execution of a significant number of elementary operations within the exciton decoherence time - a feature of fundamental importance for any useful QIP device. Sensitive detection techniques that allow for the resolution and addressing in both amplitude and phase of single quantum states are in development. Previous experimental work on individual exciton states in semiconductor nanostructures [2-4] measured the intensity of the nonlinear signal; i.e., the signal amplitude was recovered without direct access to the phase of the quantum state. The phase change by the optical Stark effect was measured indirectly in [5]. Single qubit population rotations were implemented in semiconductor quantum dots and Rabi oscillations have been demonstrated [6-9]. The observed strong damping of these oscillations is a subject of intense discussion [10-14]. The phase evolution during excitonic Rabi flopping in bulk semiconductors was recently measured [15]. Some evidence for co- herent coupling of pairs of exciton states was also found [16].

In this work we use transient four-wave mixing (FWM) detected by heterodyne spectral interferometry $[17,18]$ to recover the time-resolved state polarization in both amplitude and phase. The results are obtained using highresolution imaging, which allows the selection of an individual exciton state in a system of quantum dots. We study the state rotation for different pump detunings and show that higher excited quantum dot levels should be taken into account as a source of damping of Rabi oscillations. In an analogue to NMR, a $\pi / 2-\pi$ rotation of the state vector is performed.

The measurements were made on excitons confined by interface fluctuations in an $\mathrm{AlAs} / \mathrm{GaAs} / \mathrm{AlAs}$ quantum well $(\mathrm{QW})$ with a nominal GaAs thickness of $5 \mathrm{~nm}$. The growth and characterization of such samples is discussed in [19], and the heterodyne spectral interferometry four-wave mixing technique is described in [17]. In order to select individual states within the spatial resolution of the experiment $(0.3 \mu \mathrm{m})$, we adjusted the fractional monolayer thickness of the QW to yield a low density of islands with larger thickness [20]. Figure 1 shows the FWM spectrum for spectrally broad excitation, creating signal from any localized exciton state present in the excited sample region. The FWM is dominated by a single localized exciton state about which the excitation pulse is subsequently spectrally narrowed, allowing coherent control of the state as described below.

Having at hand a single exciton state that is resonantly driven by the two excitation pulses, we can demonstrate the possibility of a coherent manipulation of the state vector. We first analyze the case of an excitation which is exactly resonant to the transition. Figure 2(a) shows the measured FWM intensity and phase spectrally resonant to the exciton transition as a function of the pump pulse area $\Theta_{1}$. The FWM field created by the probe pulse at a delay $\tau=5 \mathrm{ps}$ after the pump pulse is proportional to the (phase- 


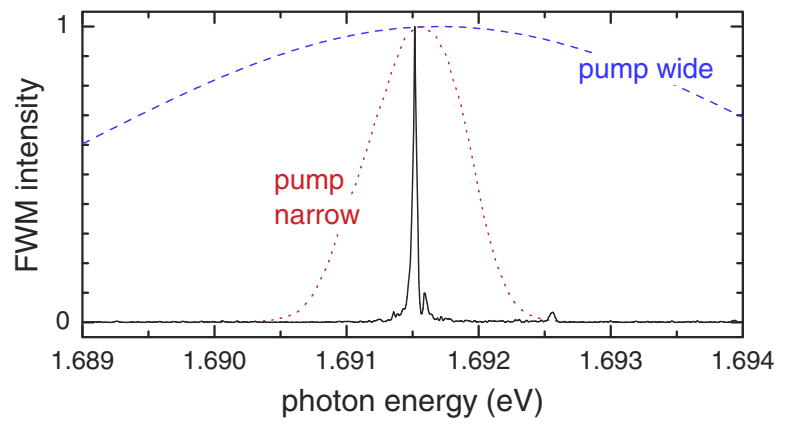

FIG. 1 (color online). Spectrally resolved FWM at $\tau=30 \mathrm{ps}$ of a $(0.5 \mu \mathrm{m})^{2}$ region of a GaAs/AlAs QW, dominated by a single localized exciton state (solid line). The energy flux per pulse 1 (2) was $1.4(2.9) \mu \mathrm{J} / \mathrm{cm}^{2}$. Also displayed are the broad excitation spectrum used here (dashed line, 0.2 ps pulse width), and the narrow one (dotted line, 2 ps pulse width) used for the measurements of the Rabi oscillations shown in Fig. 2.

conjugated) polarization created by the pump pulse. In a two-level system, Rabi oscillations would be expected as the Bloch-vector rotates [see sketches in Fig. 2(a)], with the polarization being zero both when the system is purely in the ground or the excited state, i.e., with a period of $\pi$ in the pulse area. The corresponding polarization is $\propto \sin \left(\Theta_{1}\right)$, and thus the FWM intensity $\propto \sin ^{2}\left(\Theta_{1}\right)$, shown in Fig. 2(a) as solid line. The experimental pulse area unit has been adjusted to reproduce the position of the first minimum at $\Theta_{1}=\pi$. From the focus size and the pulse duration, we estimate a transition dipole moment of 30 40 Debye. For $\Theta_{1}<1.2 \pi$, the data are in agreement with the two-level prediction. Additionally, the predicted $\pi$ jump in the phase at $\Theta_{1}=\pi$ is observed. For larger pulse areas, significant deviations from the prediction are present. Such deviations have been observed previously in a pump-probe experiment sensitive to the population inversion [6], and were interpreted as an increased dephasing rate induced by an optically created carrier density, called excitation-induced dephasing (EID), and well known in quantum wells. This mechanism was also discussed for Rabi oscillations in InGaAs quantum dots [14]. Here, we can measure the dephasing of the transition after the application of both excitation pulses using the linewidth $\gamma$ of its third-order polarization. From the missing photon echo signature in the corresponding time-resolved FWM, we can exclude a significant influence of inhomogeneous broadening on $\gamma$, due to, e.g., spectral wandering. We observe that $\gamma$ as function of pulse area $\Theta_{1}$ [see Fig. 2(a) top] is constant within error, inconsistent with the EID hypothesis. We suggest that the deviations from the twolevel result originate from the presence of near-resonant transitions to multiexcitonic states into which the system is driven by the excitation pulses - a coherent process which does not affect the dephasing of the fundamental exciton transition. To support this interpretation, we calculated the FWM intensity (see dashed line), neglecting dephasing, in presence of 3 transitions to biexcitonic states with binding
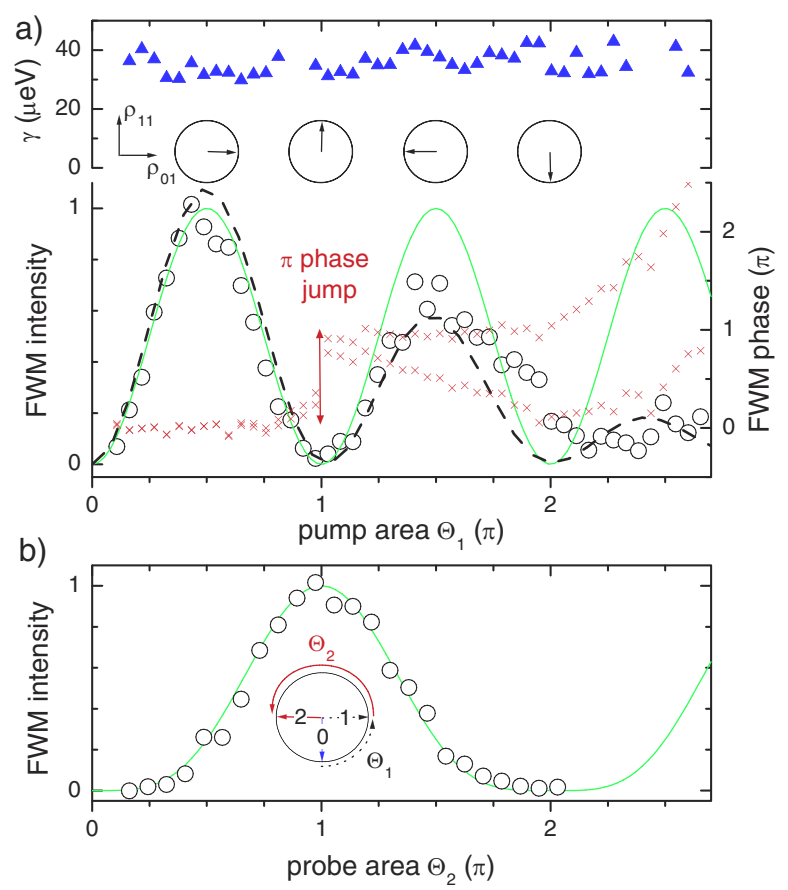

FIG. 2 (color online). FWM resonant to a single exciton state. (a) As a function of pump pulse area $\Theta_{1}$ for a probe pulse area $\Theta_{2}=\pi$. Circles: measured intensity. Solid line: expected twolevel result $\propto \sin ^{2}\left(\Theta_{1}\right)$. Dashed line: calculated FWM including multiexcitons (see text). The sketches indicate the state vectors after the pump pulse in a Bloch sphere. Crosses: measured FWM phase. The two curves indicate the limits of a systematic error due to a phase drift. Top: linewidth $\gamma$ (FWHM) of the exciton state measured in the FWM spectrum. (b) Intensity as a function of $\Theta_{2}$ for $\Theta_{1}=\pi / 2$. The solid line is the expected two-level result $\propto \sin ^{4}\left(\Theta_{2} / 2\right)$. The sketch indicates the corresponding state vector positions 0: initially, 1: after the pump, 2: after pump and probe. The energy density per excitation pulse was $2.2 \mu \mathrm{J} / \mathrm{cm}^{2}$ for $\Theta_{1,2}=\pi$, and the pulse spectrum is given in Fig. 1 .

energies of $0.5,-0.6,-0.8 \mathrm{meV}$, and transition dipole moments $30 \%$ of the exciton transition. A decay superimposed to the Rabi oscillations is observed, qualitatively reproducing the measurement. This result demonstrates the effectiveness of the suggested mechanism, while the specific biexcitonic parameters chosen are equivocal. Note that due to the strong dependence of the FWM intensity on the dipole moments and the spectrally narrow probe pulse, the FWM resonant to these biexcitonic transitions would be within the noise of the present measurement.

In FWM, the phase-conjugated third-order polarization is created from the first-order polarization by the coherent control by the probe pulse. In a two-level system, the FWM intensity created by the probe is $\propto \sin ^{4}\left(\Theta_{2} / 2\right)$ [1], with the ideal phase conjugation being achieved by rotating the Bloch vector by an angle of $\Theta_{2}=\pi$ [21]. This prediction is in quantitative agreement with the experiment [see Fig. 2(b)], and represents the verification of a basic two-pulse coherent control on the excitonic transition. This is particularly relevant since the implementation of quantum computational devices will require the ability to place 
a qubit in a given configuration, allow it to evolve (with the possible application of additional pulses to further affect the qubit state), and then the readout of the results of the evolution.

To explore the possibilities of coherent control in a two-level system by the pump pulse, we have calculated the response of a two-level system driven by a Gaussian optical pulse of field $\propto \exp \left[-\left(t / t_{0}\right)^{2}\right]$ and pulse area $\Theta_{1}$ with a detuning of $\Delta_{\omega}$ of its carrier frequency $\omega_{0}$ from the two-level resonance at $\omega_{\mathrm{X}}$. The state of the two-level system with levels 0 and 1 is described by the density matrix elements $\varrho_{i j}$, and is initially $\left(-t \gg t_{0}\right)$ in the ground state, i.e., $\varrho_{00}=1, \varrho_{01}=\varrho_{11}=0$. The properties of the final state of the two-level system $\varrho_{i j}^{(\mathrm{sp})}$ after the pulse $\left(t \gg t_{0}\right)$ are shown in Fig. 3. The occupation of the excited state $\varrho_{11}^{(\mathrm{sp})}$ shows Rabi oscillations versus $\Theta_{1}$, with a period of $2 \pi$ for $\Delta_{\omega}=0$. The polarization $\varrho_{01}^{\text {(sp) }}$ shows equivalent Rabi oscillations, which additionally include a phase evolution. At zero detuning, this leads to a $\pi$ period of $\left|\varrho_{01}^{(\mathrm{sp})}\right|^{2}$ in $\Theta_{1}$, as well as the $\pi$ phase jump at $\Theta=\pi$, which is
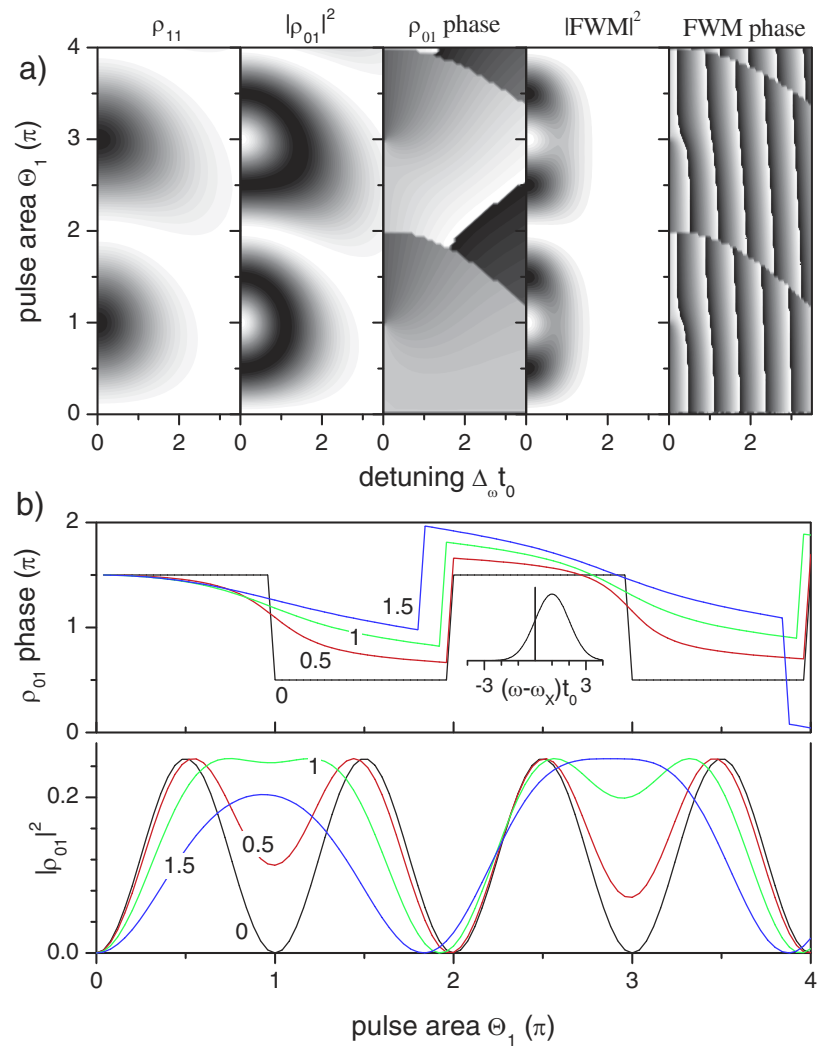

FIG. 3 (color online). Theoretical results for Rabi oscillations in a two-level system driven by Gaussian optical pulses. (a) Contour graphs of density matrix components as function of detuning and pulse area on a gray scale from white to black: occupation $\varrho_{11}$ (scale 0 to 1$)$, coherence intensity $\left|\varrho_{01}\right|^{2}$ (0 to $0.25)$, and phase $\Im\left(\ln \varrho_{01}\right)$ (0 to $\left.2 \pi\right)$. Additionally, the FWM intensity and phase are given (see text). (b) Coherence intensity and phase as function of pulse area for selected detunings $\Delta_{\omega} t_{0}$ as labeled. The inset shows the pump intensity spectrum for $\Delta_{\omega} t_{0}=1$. observable in the FWM experiment [see Fig. 2(a)]. The influence of the detuning $\Delta_{\omega}$ on the occupation of the excited state $\varrho_{11}$ is mainly of quantitative nature, showing a decrease of the oscillation amplitude and an increase of the oscillation period, according to the effective Rabi frequency $\Omega=\sqrt{\Omega_{\mathrm{R}}^{2}+\Delta_{\omega}^{2}}$ [21]. The modifications of the polarization dynamics are more pronounced. In the presence of detuning the top of the Bloch sphere is not reached, so that the polarization does not vanish for $\pi$ pulse area. The phase dynamics accordingly does not have a $\pi$ jump at $\Theta_{1}=\pi$, but shows a smooth phase evolution. For larger detuning, even the equator of the Bloch sphere is no longer reached, so that only a single maximum is observed, similar to the dynamics of the population density $\varrho_{11}^{(\mathrm{sp})}$. To compare these results with the measured FWM, we calculated the response of the two-level system to a sequence of two pulses, where the second pulse of area $\Theta_{2}=\pi$ and phase $\varphi_{2}$ has a delay of $5 t_{0}$. The FWM polarization $\varrho_{01}^{(\mathrm{FWM})}$ was then determined as the component of $\varrho_{01}$ after the second pulse with the phase dependence $e^{i\left(2 \varphi_{2}-\varphi_{1}\right)}$, where $\varphi_{1}$ is the phase of the first pulse. The resulting FWM, given in the rightmost 2 panels of Fig. 3(a), shows a much stronger dependence on the detuning compared to $\varrho_{01}^{(\mathrm{sp})}$. However, the dependence on $\Theta_{1}$ is conserved, i.e., $\varrho_{01}^{(\mathrm{FWM})}=C\left(\Theta_{2}, \Delta_{\omega}\right)\left(\varrho_{01}^{(\mathrm{sp})}\right)^{*}$, with a complex function $C\left(\Theta_{2}, \Delta_{\omega}\right)$. This justifies the direct comparison of measured FWM and $\varrho_{01}^{(\mathrm{sp})}$ in Fig. 2(a).

The predicted behavior versus detuning shows a way to coherent phase control, which we have implemented by shifting the center wavelength of all pulses. Figure 4 shows the $\Theta_{1}$ dependence of the FWM signal (symbols) for different detunings. The calculated FWM of a two-level system (lines) is in quantitative agreement for $\Theta_{1}<\pi$, while for larger areas deviations are observed as discussed
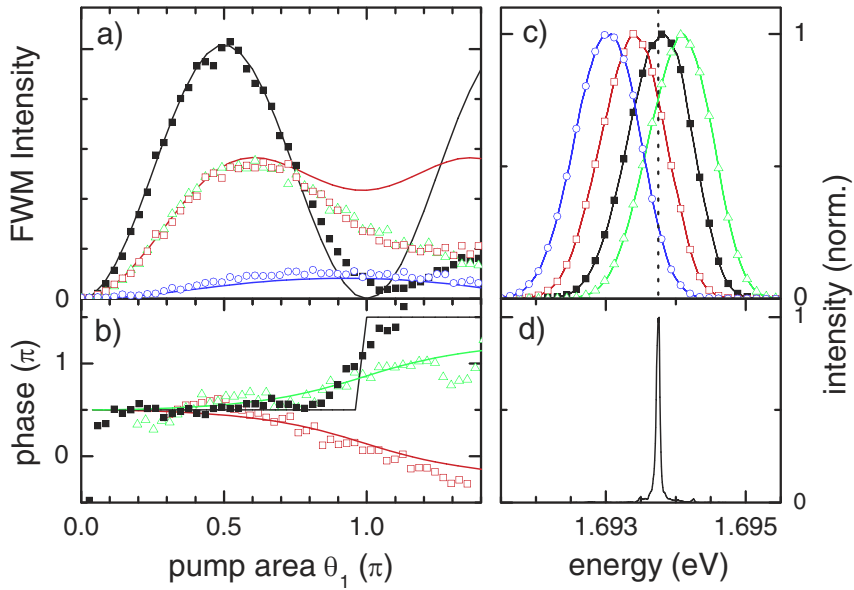

FIG. 4 (color online). Rabi oscillations of a single excitonic state vs detuning. FWM intensity (a) and phase (b) as function of $\Theta_{1}$ for $\Theta_{2}=\pi$ and various detunings $\hbar \Delta_{\omega}\{-0.73,-0.33$, $+0.02,+0.34\} \mathrm{meV}$. Solid lines: calculated FWM. The corresponding excitation spectra are given in (c). The FWM spectrum at $\Theta_{1}=\pi / 2$ (pulse energy density $2.6 \mu \mathrm{J} / \mathrm{cm}^{2}$ ) is given in (d). 


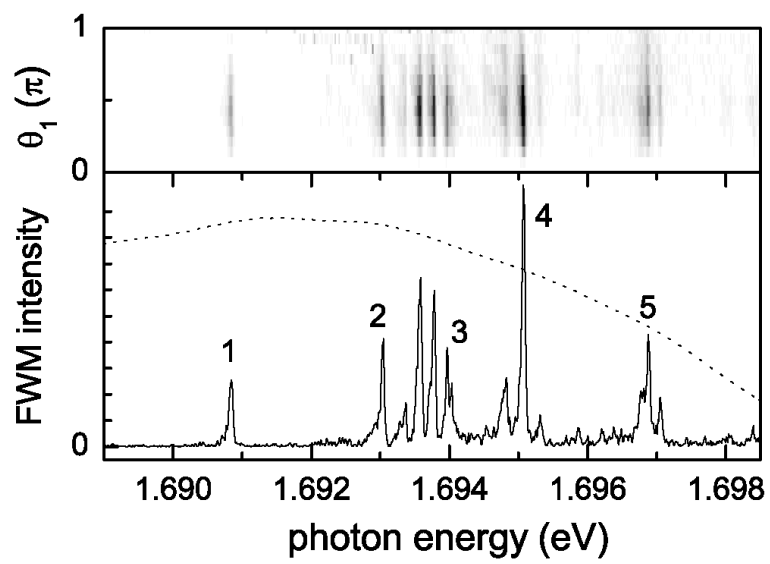

previously. With increasing detuning, a reduced maximal polarization and a finite polarization at $\Theta_{1}=\pi$ is found as predicted. The measured phase responses for zero and $\pm 0.33 \mathrm{meV}$ detuning are given in Fig. 4(b). They demonstrate a phase control using pulse area and detuning.

To evaluate the feasibility of a simultaneous application of a gate operation on a set of two-level systems, we controlled a small ensemble of transitions located within the spatial resolution of $0.3 \mu \mathrm{m}$ by a spectrally broad pulse. No mutual coherent coupling between the transitions was present [22]. The resulting $\Theta_{1}$ dependence of the FWM intensity (see Fig. 5) of the various transitions is very similar. This observation shows the possibility of a simultaneous application of a gate operation on a set of two-level systems, enabling parallel processing in a quantuminformation device.

In conclusion, we have presented several aspects of the coherent control and readout of individual exciton states using resonant coherent control and polarization readout by transient four-wave mixing. We find that excitationinduced dephasing is negligible in the investigated system, enabling the implementation of complex quantum control beyond the one shown in this work. Coherent control with nonresonant excitation pulses was demonstrated, opening the possibility of controlling both the amplitude and the phase of the quantum state.

The sample was grown by K. Leosson and J. Riis Jensen at the III-V Nanolab, a joint laboratory between Research Center COM and the Niels Bohr Institute, Copenhagen University. This work was supported by the German Science Foundation (DFG) within the Grant No. WO477/ 14 and GRK 726.

[1] E. L. Hahn, Phys. Rev. 80, 580 (1950).

[2] J. Guest, T. H. Stievater, G. Chen, E. A. Tabak, B. G. Orr, D. G. Steel, D. Gammon, and D. S. Katzer, Science 293, 2224 (2001).

[3] T.H. Stievater, X. Li, D. G. Steel, D. Gammon, D. S. Katzer, and D. Park, Phys. Rev. B 65, 205319 (2002).

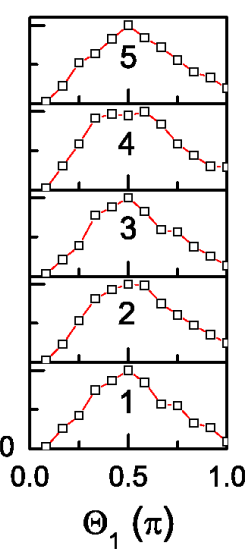

FIG. 5 (color online). FWM intensity of an ensemble of exci>ton states. Bottom: spectrum at $\Theta_{1}=\pi / 2$. The excitation spectrum is given as dotted line. Top: spectrum as function of $\Theta_{1}$ on a linear gray scale from 0 (white) to black. Right: intensity of the individual transitions (as labeled in the spectrum) vs pulse area.
[4] T. Guenther, C. Lienau, T. Elsaesser, M. Glanemann, V. Axt, T. Kuhn, S. Eshlaghi, and A. Wieck, Phys. Rev. Lett. 89, 057401 (2002).

[5] T. Unold, K. Mueller, C. Lienau, T. Elsaesser, and A. D. Wieck, Phys. Rev. Lett. 92, 157401 (2004).

[6] T. H. Stievater, X. Li, D. G. Steel, D. S. Katzer, D. Park, C. Piermarocchi, and L. Sham, Phys. Rev. Lett. 87, 133603 (2001).

[7] H. Kamada, H. Gotoh, J. Temmyo, T. Tagakahara, and H. Ando, Phys. Rev. Lett. 87, 246401 (2001).

[8] H. Htoon, T. Tagakahara, D. Kulik, O. Baklenov, A. L. Holmes, and C. K. Shih, Phys. Rev. Lett. 88, 087401 (2002).

[9] A. Zrenner, E. Beham, S. Stufler, F. Findeis, M. Bichler, and G. Abstreiter, Nature (London) 418, 612 (2002).

[10] P. Borri, W. Langbein, S. Schneider, U. Woggon, R. L. Sellin, D. Ouyang, and D. Bimberg, Phys. Rev. B 66, 081306(R) (2002).

[11] J. Förstner, C. Weber, J. Danckwerts, and A. Knorr, Phys. Rev. Lett. 91, 127401 (2003).

[12] P. Machnikowski and L. Jacak, Phys. Rev. B 69, 193302 (2004).

[13] L. Besombes, J. J. Baumberg, and J. Motohisa, Phys. Rev. Lett. 90, 257402 (2003).

[14] Q. Q. Wang, A. Muller, P. Bianucci, E. Rossi, Q. K. Xue, T. Takagahara, C. Piermarocchi, A. H. MacDonald, and C. K. Shih, Phys. Rev. B 72, 035306 (2005).

[15] N.C. Nielsen, T. H.Z. Siederdissen, J. Kuhl, M. Schaarschmidt, J. Förstner, A. Knorr, and H. Giessen, Phys. Rev. Lett. 94, 057406 (2005).

[16] T. Unold, K. Mueller, C. Lienau, T. Elsaesser, and A. D. Wieck, Phys. Rev. Lett. 94, 137404 (2005).

[17] W. Langbein and B. Patton, Phys. Rev. Lett. 95, 017403 (2005).

[18] W. Langbein and B. Patton (to be published).

[19] K. Leosson, J. R. Jensen, W. Langbein, and J. M. Hvam, Phys. Rev. B 61, 10322 (2000).

[20] V. Savona, W. Langbein, and G. Kocherscheidt, Phys. Status Solidi C 1, 501 (2004).

[21] L. Allen and J.H. Eberly, Optical Resonance and TwoLevel Atoms (Wiley, New York, 1975).

[22] The coupling was determined using the delay-time dependence of the FWM, as described in [23].

[23] B. Patton, U. Woggon, and W. Langbein, in QELS 2005, OSA Technical Digest (Optical Society of America, Washington, DC, 2005). 